\title{
Effect of the mutarotase on the analytical behaviour of modified carbon paste type glucose biosensor
}

\author{
S. Gueguen, M. Boujtita and N. El Murr
}

Groupe Électrochimie - Laboratoire d'Analyse Isotopique et Électrochimique de Métabolismes (UPRESA, CNRS, 6006) Faculté des Sciences et des Techniques, 2, rue de la Houssinière. B.P 92208, 44322 Nantes Cedex 03 France

\begin{abstract}
A bi-enzymatic carbon paste electrode modified with glucose oxidase and mutarotase was prepared and compared in terms of analytical performance (sensitivity, repeatability and accuracy) to a mono-enzyme modified electrode with glucose oxidase. Using the chronoamperometric technique compared the analytical parameters of both preparations. The bi-enzymatic electrode response showed that the mutarotase addition into the carbon paste electrode in the presence of GOD improved the sensitivity towards glucose but both repeatability and accuracy were affected.
\end{abstract}

sisted of mixing together carbon and pasting liquid, has been widely investigated [13]; new modified electrodes appeared with other components like enzymes [14], electrochemical mediator [15] or both [16-20] mixed in the carbon paste.

In our laboratory, we have developed carbon paste electrode (CPE) for glucose measurements, suitable for industrial needs in terms of accuracy and repeatability [21]. We are now investigating the possibility of the fabrication of a multi-enzyme CPE for sucrose measurement which should include in its bulk glucose oxidase, mutarotase and invertase. Before the accomplishment of the tri-enzyme CPE, our first interest was to study the effect of the incorporation of mutarotase on the signal response of the biosensor in terms of sensitivity, repeatability and accuracy, when only glucose is detected.

\section{Experimental}

\section{Reagents}

Glucose oxidase (E.C. 1.1.3.4, 258 I.U mg-1) and mutarotase (E.C. 5.1.3.3, 2140 I.U mg-1) were purchased from Biozyme, Great Britain. The ferrocene was obtained from Strem Chimicals, USA, Glutaraldehyde from Sigma, USA, Bovine Serum Albumin (BSA) and castor oil from Merck, Germany and carbon powder from Carbone Lorraine, France. Stock solutions of glucose were prepared by dissolving glucose anhydrous (Fluka, reagent-grade chemicals) in $0.1 \mathrm{M}$ phosphate buffer $(\mathrm{pH} 7.4)$ containing $0.1 \%$ of triton (X-100, Labogros, France). All glucose measurements were carried out at room temperature $\left(20-23^{\circ} \mathrm{C}\right)$.

\section{Electrode preparation}

Among these biosensors, amperometric glucose electrodes towards this substrate [1]. Most of the electrodes were first based on co-immobilised enzyme membrane [2], [3] in connection with a metal, a glassy carbon or a Clark electrode. Others enzymes ( $\beta$-galactosidase, mutarotase, invertase) could be added to get multi-enzyme membranes able to detect more complex carbohydrates, giving by enzymatic hydrolysis, glucose [4-11]. The carbon paste [12], which con-
Two batches of Carbon Paste Electrode (CPE) modified with Glucose Oxidase (GOD) or with GOD and mutarotase (MUT), both containing a redox mediator (ferrocene) were prepared. $5 \mathrm{mg}$ of BSA were dissolved in $0.667 \mathrm{~mL}$ of distilled water stirred at room temperature. A given amount of GOD corresponding to 50000 International Units (I.U) was 
then added. 5000 I.U of MUT were added for one of the batch preparation. Reticulation of enzymes was performed by adding $0.330 \mathrm{~mL}$ of glutaraldehyde solution $(0.25 \%$ in distilled water) to the previous enzymatic mixture that was kept under stirring for $10 \mathrm{~min}$ at room temperature. Finally, the carbon powder $(1 \mathrm{~g})$ was added. In order to improve the homogeneity of this preparation, the carbon powder was added while stirring the enzymatic mixture and kept under these conditions for 20 minutes. After homogenisation, the resulting mixture was freeze-dried.

The resulting GOD or GOD-MUT modified carbon powder was mixed by hand in a mortar with a given amount of castor oil (23.23\% - final oil percentage in the paste) already containing ferrocene $(0.34 \%)$. A plastic tube with internal diameter of $0.3 \mathrm{~cm}$ was then filled up with the paste obtained. Inserting a graphite rod at one end of the plastic tube provided electrical contact.

\section{Measurement procedure}

All measurements were performed using the "Multicapteur MC2" developed and commercialised by Gwent Sensors Limited (UK). This detection apparatus consists of an electronic unit and of an electrode handle containing a working electrode (i.e. the previous enzymatic paste filled in a plastic cartridge) inserted in concentric quasi-reference platinum electrode. A circular surface $\left(7 \mathrm{~mm}^{2}\right)$ at the bottom of the cartridge is in contact with the glucose solution during the analysis whereas a metal piston inserted at the top of the cartridge in contact with the graphite rod permits the flow of the current through the electronic unit. The electronic activation of the piston induces the expelling of a small amount of paste, which makes it possible to restore an identical surface by polishing the working electrode after each measurement.

The applied potential is $+0,9 \mathrm{~V}$ versus $\mathrm{Pt}$ (quasi-reference electrode) and the measurement time is of 20 seconds. In order to provide significant results, each measurement was performed at least three times.

\section{Results and discussion}

The glucose electrode based on GOD and $\mathrm{FcH}$ as redox mediator uses the following consecutive reactions to shuttle electrons between the reduced enzyme and the electrode surface:

\section{With or without mutarotase}

(1) $\alpha$-glucose $(1 / 3)$

$\beta$-glucose

2) GOD (ox) $+\alpha$-glucose

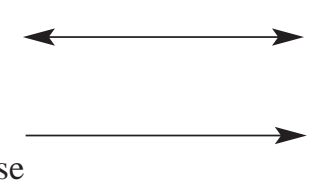

GOD (red) + gluconic acid $+2 \mathrm{H}^{+}$

\section{(3) GOD (red) $+2 \mathrm{FcH}^{+}$}

(4) $2 \mathrm{FcH}$

$$
\text { GOD (ox) }+2 \mathrm{FcH}
$$

$$
2 \mathrm{FcH}^{+}+2 \mathrm{e}-
$$

The first step of the measurement is the oxidation of $\mathrm{FcH}$ at the electrode surface in order to electrochemically produce ferricinium cation $\mathrm{FcH}^{+}$. In the presence of glucose, $\mathrm{FcH}^{+}$is involved in the regeneration of the enzymatic active form of GOD leading to the catalytic current. The intensity of the resulting catalytic current depends on the glucose concentration. In this study, as was described in the experimental section, both GOD and $\mathrm{FcH}$ were incorporated into the carbon paste. However such an electrode is able to detect only the $\beta$-anomer of the glucose because of the specificity of the glucose oxidase. It is well known that the mutarotation of $\alpha$-glucose into its $\beta$-anomer is very slow and the equilibrium between the two anomers needs a long time to be obtained. Therefore, at room temperature and in the absence of mutarotase, reaction (1) is very slow. So no or negligible effect of the reaction (1) could be observed on the electrochemical signal when the measurement time is fixed at $20 \mathrm{~s}$.

In order to study the effect of the mutarotation reaction on the electrochemical signal, two GOD/FcH modified electrodes were prepared and tested in the presence and the absence of mutarotase. The mutarotase was incorporated into the carbon paste in the presence of GOD. Figure 1 displays the comparison of calibrations curves obtained with the two resulting electrodes. The electrode response was examined between 0.5 and $16.7 \mathrm{mM}$ of glucose at $\mathrm{pH} 7.2$ with a measurement time of $20 \mathrm{~s}$. It has been suggested that total glucose detection $(\alpha+\beta)$ was limited by the mutarotation of $\alpha$ glucose into its $\beta$-anomer and considered as the rate-limiting step [22]. In our case, the highest sensitivity was obtained with the addition of mutarotase into the electrode, indicating that in the presence of mutarotase the chemical equilib-

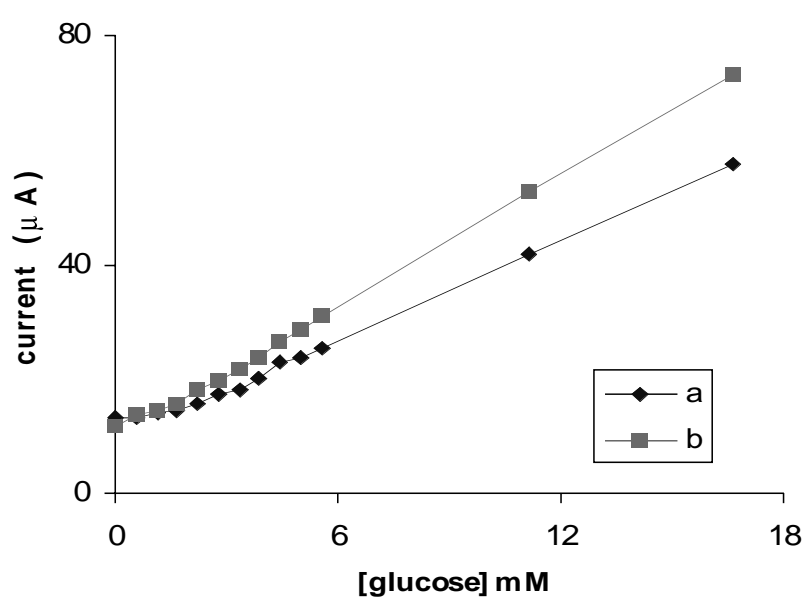

Figure 1. Effect of mutarotase on the electrochemical response of modified carbon paste electrode with GOD, FcH (a) and GOD, $\mathrm{FcH}$ and mutarotase (b). 
Table I. Estimation of the glucose detection in the presence of mutarotase and glucose oxidase at the carbon paste electrode surface.

The equation $\Delta \mathrm{l}_{\text {GOD CPE }}=4.26[\beta$-glucose $]-3.65$ with $r^{2}=0.9993$ corresponds to the calibration curve obtained in the absence of mutarotase.

\begin{tabular}{|c|c|c|c|c|c|}
\hline $\begin{array}{c}\text { [glucose] } \\
\mathrm{mM}\end{array}$ & $\begin{array}{c}\text { [ } \beta \text {-glucose }] \\
m M\end{array}$ & $\begin{array}{c}\Delta l^{*} G O D C P E \\
\mu A\end{array}$ & $\begin{array}{c}\Delta l{ }^{*} G O D-M U T \\
C P E \mu A\end{array}$ & $\begin{array}{c}{[\beta \text {-glucose }]^{* *}} \\
\text { corresponding values } \\
m M\end{array}$ & $\begin{array}{c}\text { Recovery vs. total } \\
\text { glucose } \\
\%\end{array}$ \\
\hline 1.7 & 1.3 & 1.49 & 4.02 & 1.8 & 108 \\
\hline 2.2 & 1.5 & 2.53 & 6.38 & 2.3 & 106 \\
\hline 2.8 & 1.9 & 4.02 & 8.25 & 2.8 & 101 \\
\hline 3.3 & 2.2 & 5.09 & 10.19 & 3.2 & 97 \\
\hline 3.9 & 2.6 & 6.86 & 12.23 & 3.7 & 96 \\
\hline 4.4 & 2.9 & 9.67 & 14.97 & 4.4 & 98 \\
\hline 5.0 & 3.3 & 10.60 & 16.74 & 4.8 & 96 \\
\hline 5.6 & 3.7 & 12.20 & 19.34 & 5.4 & 97 \\
\hline 11.1 & 7.4 & 28.49 & 41.15 & 10.5 & 95 \\
\hline 16.7 & 11.1 & 44.19 & 61.67 & \multicolumn{2}{|c|}{ Value out of the regression range } \\
\hline
\end{tabular}

${ }^{*} \Delta \mathrm{I}=\left(\mathrm{I}_{\mathrm{k}}-\mathrm{I}_{\mathrm{d}}\right)$ where $\mathrm{I}_{\mathrm{k}}$ is the catalytic current and $I_{d}$ the diffusional current.

** Values calculated using the equation of the curve obtained without mutarotase for $\Delta \mathrm{I}_{\mathrm{GOD}-\mathrm{MUT}} \mathrm{CPE}$.

rium sets up almost instantaneously as the $\beta$-glucose is consumed. When the electrode contains only glucose oxidase, the current measured depends only on the diffusion of the $\beta$-anomer [23]. Indeed, as it has been mentioned previously, in the absence of mutarotase the equilibrium is very slow to set up and only the $\beta$-form is detected: the current is then proportional to the amount of $\beta$-glucose in solution i.e. $66,7 \%$ of total glucose. In this case, the decrease in the $\beta$ glucose concentration in the vicinity of the electrode surface causes a glucose gradient and only $\beta$-glucose could react at the electrode surface. However, when both mutarotase and glucose oxidase are incorporated in the carbon paste, higher electrode response was observed (Fig. 1), which is attributed to a mutarotation phenomenon producing $\beta$-glucose from $\alpha$ glucose during the measurement time. Taking into account that the GOD recognises only $\beta$-glucose; the signal intensity was presented versus $\beta$-glucose that corresponds to $66.7 \%$ of the total glucose concentration. Thereby, the recovery rate of total mutarotation of glucose could be easily estimated. This estimation is presented in table I. It can be clearly observed, by calculating a regression between the current obtained by the GOD CPE and the corresponding values of $\beta$-glucose and incorporating the current values of GODMUT CPE, that a larger part of $\alpha$-glucose is transformed and then detected at the electrode surface during the measurement time. This seems to indicate that the reaction rate of mutarotation is very quick and that the current of the bienzymatic electrode could be controlled by the diffusion of both $\alpha$ and $\beta$-glucose from the bulk solution to the electrode surface. Under these experimental conditions, it seems that the electrode could detect both the $\alpha$ and the $\beta$ anomers.

The mutarotase effect on the analytical performance such as sensitivity, repeatability and accuracy was also investi- gated. The reproducibility of the electrode preparation was examined by comparing a set of five electrodes in presence and in absence of mutarotase. Two series of a twelve-measurement calibration were carried out on each electrode. The slopes and intercepts values obtained from different calibration curves were used as indicators for the evaluation of the reproducibility preparation of the electrode. Simple mean and variance tests [24] were applied on these data to assess in a statistical way, whether or not any significant difference in terms of calibration exists between both types of electrode. The results are shown in table II. It can be observed that the presence of mutarotase does not only increase the sensitivity but also increases the value of the intercept. The standard deviations $\sigma$ associated with intercept and slope values are found to be also affected by the presence of mutarotase in the GOD/FcH modified carbon paste electrode; $\sigma$ increases roughly by a factor of 2 for the slope (a) and by a factor of 3 for the intercept (b).

Table II also displays a significant difference in the sensitivity variance between the $\mathrm{GOD} / \mathrm{FcH}$ electrode prepared with and without mutarotase. The Fisher test confirms different variance behaviour between the GOD and the GODMUT CPE. In order to compare in a more usual way the difference of reproducibility between both batches, we chose as indicator the relative standard deviation (RSD) of each parameter. Though a clear difference of variance was observed by applying a Fisher test, the RSD relating to the sensitivity of the GOD-MUT electrode reproducibility is acceptable $(1.8 \%)$ if compared to the one calculated for the GOD electrode (1.2\%). However, the reproducibility is quite affected when comparing the intercepts of both batches. The RSD of the intercept associated with the GOD-CPE batch is 9.0\% whereas the one associated with the GOD-MUT batch 
Table II. Effect of mutarotase on the parameters (slope: a and intercept : b) of the calibration curve equation. $\mathbf{a}=$ slope of the calibration curve $=$ sensitivity (expressed in $u A \cdot \mathrm{mM}^{-1}$ )

\begin{tabular}{lccccc}
\hline & Mean $^{*}$ & $\begin{array}{c}\text { Standard } \\
\text { deviation } \sigma\end{array}$ & $\begin{array}{c}\text { Relative Standard } \\
\text { Deviation }(R S D) \%\end{array}$ & $\begin{array}{c}\text { Means }^{* *} \\
\text { equality } t \text { test }\end{array}$ & $\begin{array}{c}\text { Variances }^{* *} \\
\text { equality } F \text { test }\end{array}$ \\
\hline GOD & 2.69 & 0.0324 & 1.2 & & 4.69 \\
GOD-MUT & 3.87 & 0.0702 & 1.8 & 46.03 & 4.69 \\
\hline
\end{tabular}

* Mean of 10 values

** Probability of the test $<1 \%$

b = intercept of the calibration curve

\begin{tabular}{lccccc}
\hline & Mean $^{*}$ & $\begin{array}{c}\text { Standard } \\
\text { deviation } \sigma\end{array}$ & $\begin{array}{c}\text { Relative Standard } \\
\text { Deviation }(R S D) \%\end{array}$ & $\begin{array}{c}\text { Means }{ }^{* *} \\
\text { equality } t \text { test }\end{array}$ & $\begin{array}{c}\text { Variances }^{* *} \\
\text { equality } F \text { test }\end{array}$ \\
\hline GOD & 7.03 & 0.63 & 9.0 & & 9.58 \\
GOD-MUT & 9.32 & 1.95 & 20.9 & 3.35 & 9.58 \\
\hline
\end{tabular}

* Mean of 10 values

** Probability of the test $<1 \%$

The coefficient $t$ is given by the equation (1) :

$\mathrm{t}=\frac{\text { Mean }_{1}-\text { Mean }_{2}}{\sigma_{\mathrm{c}}\left(1 / \mathrm{n}_{1}+1 / \mathrm{n}_{2}\right)^{0.5}}$

$\mathrm{n}_{1}$ : number of values for the Mean $_{1}$ calculation

$\mathrm{n}_{2}$ : number of values for the Mean $\mathrm{n}_{2}$ calculation

$\sigma_{\mathrm{c}}$ : pool standard deviation values given by the equation (2)

$\sigma_{c}^{2}=\frac{\left(n_{1}-1\right) \sigma_{1}^{2}+\left(n_{2}-1\right) \sigma_{2}^{2}}{\left(n_{1}+n_{2}-2\right)}$

The ratio $F$ is given by the equation (3) :

(3) $F=\sigma_{1}^{2} / \sigma_{2}^{2}$

is $20.9 \%$. This last result seems to indicate a more variable base line current in the carbon paste when mutarotase is incorporated. The addition of mutarotase into the carbon paste provides higher sensitivity but also higher and more variable base line current, which affects the repeatability of the intercept calibration parameter.

The signal dispersion of the electrode response was also examined by estimating the difference between the maximum and minimum current value for a succession of 25 measurements at the same concentration in the middle of the linear range $(8.3 \mathrm{mM})$. Five electrodes were checked for both types of CPE and two series of 25 measurements were carried out on each electrode. The results are shown in table III. As expected, the average current dispersion for the GOD-MUT $(3.95 \mu \mathrm{A})$ CPE is more important, almost twice the value of the one for GOD CPE $(2.35 \mu \mathrm{A})$. Considering the variance and the Fisher test $(\mathrm{F}=6.06)$, we noticed a significant difference for current dispersion inside the same fabrication batch of GOD-MUT electrode. The standard deviation for GOD CPE is 0.93 whereas the one for GODMUT CPE is 2.39. The presence of mutarotase inside the carbon modified paste affects the repeatability of the mea- surements. This could be due to a non-homogeneous distribution of mutarotase at the CPE surface. Indeed, a very low concentration of mutarotase was present in the paste. This rate was roughly evaluated at $0.2 \%$ (instead of $20 \%$ for the GOD). Though the mutarotase concentration is very low, it was found sufficient to reach the maximum current. Significant increases of the amount of mutarotase in the CPE did not improve the repeatability of the measurement.

Table III. Signal dispersion ( $\mu$ A ) of GOD and GOD-MUT CPE.

\begin{tabular}{lcccc} 
& Mean $^{*}$ & $\begin{array}{c}\text { Standard } \\
\text { deviation } \sigma\end{array}$ & $\begin{array}{c}\text { Means }^{* *} \\
\text { equality } \\
\text { test } t\end{array}$ & $\begin{array}{c}\text { Variances }^{* *} \\
\text { equality } \\
\text { test } F\end{array}$ \\
\hline GOD & 2.15 & 0.93 & 2.30 & 6.06 \\
GOD-MUT & 3.95 & 2.39 & & \\
\hline
\end{tabular}

* 10 series of 25 successive measurements, $8.3 \mathrm{mM}$

** Probability of the test $<5 \%$ 
Table IV. Distribution of the concentration error percentages* for GOD and GOD-MUT electrodes for 250 measurements.

\begin{tabular}{|c|c|c|c|}
\hline & $C_{\text {error }} \%>3 \%$ & $C_{\text {error }} \%>5 \%$ & $C_{\text {error }} \%>10 \%$ \\
\hline GOD** & $8,40 \%$ & $0,80 \%$ & $0 \%$ \\
\hline GOD MUT ${ }^{\star \star *}$ & $49,80 \%$ & $23,89 \%$ & $4,86 \%$ \\
\hline
\end{tabular}

* The concentration error percentage is calculated using the following formula

$\mathrm{C}_{\text {error }} \%=$ (estimated [glucose] - real[glucose] $) /$ real[glucose] ${ }^{*} 100$ real [glucose] is given by a standard method i.e. weighing

** 250 measurements

*** 247 measurements

The mutarotase effect on the accuracy of the electrode measurement was also examined by calculating the error percentage on glucose concentration. We can easily expect, considering the previous results that the GOD-MUT electrodes will give estimated glucose concentration values less accurate than GOD electrodes. Table IV gives the concentration error percentages distribution for GOD and GOD-MUT electrodes calculated on a representative sample of 250 measurements. As expected, the concentration error percentages are higher for the GOD-MUT CPE. While more than $90 \%$ of the measurements of the GOD electrodes are included in the $3 \%$ acceptable error limit, almost more than half the measurements of the bi-enzymatic electrodes have been found to exceed the $3 \%$ error limit. Measurements done by the bienzymatic electrode are less repeatable and less accurate than measurements done by the GOD enzymatic electrode.

\section{Conclusion}

A significant improvement of the sensitivity was obtained when the mutarotase was confined into the carbon paste in the presence of GOD and $\mathrm{FcH}$. However, the analytical performance is dramatically affected in terms of repeatability and accuracy. This result could be due to the decrease in the mutarotase activity of the electrode during the preparation of the modified carbon paste, leading to the irreproducible surface in terms of enzymatic units. The improvement in the repeatability of such a bi-enzyme electrode could be achieved by improving the stability of mutarotase. We are currently working in this direction.

\section{References}

1. Guilbaut, G.G.; Lubrano, G.J. Analytica Chimica Acta 1973, 64, 439-455.

2. Thévenot, R.; Strenberg, R.; Coulet, P.R.; Laurent, J.; Gautheron, D.C. Analytical Chemistry 1979, 51(1), 96-99.

3. Kulys, J.J.; Pesliakiene, M.V.; Samalius, A.S. Bioelectrochemistry and Bioenergetics 1981, 8, 81-88.

4. Cordonnier, M., Lawny, F.; Chapot, D.; Thomas, D. Febs Letters 1975, 59(2), 263-267.

5. Neubauer, A.; Hòdl, C.; Pum, D.; Sleytr, U.B. Analytical letters 1984, 27(5), 849-865.

6. Scheller, F.; Karsten, C.H. Analytica Chimica Acta 1983, 155 , 29-36.

7. Watanabe, E.; Tagaki, M.; Takei, S.; Hoshi, M., Shu-Gui, C. Biotechnology and Bioengineering 1991, 38, 99-103.

8. Nabi Rahni, M.A.; Lubrano, G.J.; Guilbault, G.G. J. Agric. Food Chem 1987, 35, 1001-1004.

9. Xu, Y.; Guilbault, G.; Kuan, S. Analytical Chemistry 1989, 61, 782-784.

10. Satoh, I.; Karube, I.; Suzuki, S. Biotechnology and Bioengineering 1976, 18, 269-272.

11. Popp, J.; Silber, A.; Braeuchle, C.; Hampp, N. Biosensors and Bioelectronics 1995, 10, 243-249.

12. Adams, R.N. Analytical Chemistry 1958, 30, 1576.

13. Gorton, L. Electroanalysis 1995, 7(1), 23-45

14. Yao, T.; Musha, S. Anal. Chim. Acta 1979, 110, 203.

15. Schultz, F.A.; Kuwana, T. J. Electroanal. Chem. 1965, 10, 95103.

16. Amine, A.; Kauffmann, J.-M.; Patriarche, G.J. Analytical Letters 1991, 24(8), 1293-1315.

17. Amine, A., Kauffmann, J.-M. Bioelectrochemistry and Bioenergetics 1992, 28, 117-125.

18. Kulys, J., Hansen, H.E. Anal. Chim. Acta 1995, 303, 285-294.

19. Boujtita, M.; El Murr, N. J. Food Sci. 1995, 60(1), 201-204.

20. Boujtita, M.; Chapleau, M.; El Murr, N. Electroanalysis 1996, 8(5), 485-488

21. El Murr N.; Slilam, M. (Brevets): Fr 2630 546; EU 409 896; JP 504 914/89; US 527208722.

22. Abdul Hamid, J.; Moody, G.J.; Thomas, J.D.R. Analyst 1988, 113, 81-85.

23. Rondeau, A. Effect of the enzyme mutarotase in solution during the glucose determination by a single enzyme-mediator based electrode (to be published).

24. Miller, J.C.; Miller, J.N. Statistics for Analytical Chemistry; 3rd edition, Ellis Horwood Limited, 1993. 